\title{
Nonlinearity effects in the kicked oscillator
}

\author{
Roberto Artuso ${ }^{a, b, c, \dagger}$ and Laura Rebuzzini ${ }^{a}$ \\ ${ }^{(a)}$ Centre for Nonlinear and Complex Systems and Dipartimento di Scienze Chimiche, Fisiche e Matematiche, Università \\ dell'Insubria, Via Valleggio 11, 22100 Como, Italy \\ ${ }^{(b)}$ Istituto Nazionale di Fisica della Materia, Unità di Como, Via Valleggio 11, 22100 Como, Italy \\ ${ }^{(c)}$ Istituto Nazionale di Fisica Nucleare, Sezione di Milano, Via Celoria 16, 20133 Milano, Italy \\ (†) email address; roberto.artuso@uninsubria.it
}

The quantum kicked oscillator is known to display a remarkable richness of dynamical behaviour, from ballistic spreading to dynamical localization. Here we investigate the effects of a Gross Pitaevskii nonlinearity on quantum motion, and provide evidence that the qualitative features depend strongly on the parameters of the system.

PACS numbers:05.45.-a

The dynamical behavior of quantized area-preserving maps has proved to be one the most relevant field in the discipline of quantum chaos (see [1]): in particular the discovery of quantum dynamical localization [2], namely the quantal suppression of classical deterministic diffusion, has provoked a vast amount of theoretical and experimental work. The paradigmatic example in this field is the quantum kicked rotator (see [3]), obtained upon quantization of the classical standard map [4]. From a classical point of view the system is of KAM nature: for small values of the stochasticity parameter global transport is inhibited by invariant curves: once the last invariant torus is destroyed, transport properties abruptly change, and, in typical situations of strong chaos, there is a diffusive spreading in momentum [4.5], which characterizes also the quantum motion for times shorter than the break-time $t_{b}$, where quantum localization regime sets in and the momentum spreading is suppressed [6]. We remark that this picture is valid for generic values of the effective Planck's constant: quantum resonant motion, characterized by ballistic spreading, appears when $\hbar$ assumes rational values [2,8].

Another example of a quantum system originating from a two dimensional area preserving map is the kicked harmonic oscillator (see 9011] and references therein): the classical hamiltonian is

$$
\mathcal{H}(p, x, t)=\frac{1}{2 m_{0}} p^{2}+\frac{m_{0}}{2} \omega_{0}^{2} x^{2}+\varepsilon \cos \left(k_{0} x\right) \delta_{T_{0}}(t)
$$

where the time dependence is through the periodic delta function

$$
\delta_{T_{0}}(t)=\sum_{m=-\infty}^{\infty} \delta\left(t-T_{0} m\right)
$$

By rescaling variables $\tilde{x}=k_{0} x$ and $\tilde{t}=\omega_{0} t$ we realize that dynamics is only dependent on the parameters

$$
\mathcal{K}=\frac{\varepsilon k_{0}^{2}}{m_{0} \omega_{0}} \quad T=T_{0} \omega_{0}
$$

In particular the resonant case $(T=2 \pi p / q)$ is characterized by the presence of a stochastic web (for arbitrarily small values of $\mathcal{K}$ ) supporting unbounded transport [12,13, while in the non-resonant case a threshold $\mathcal{K}\left(E_{0}\right)$ exists below which unbounded transport is not sustained [14.

The kicked harmonic oscillator has been proposed as a model of different physical phenomena: from electronic transport in semiconductor superlattices [15], to ion traps [16. In the latter case the harmonic potential is representative of the ion trap, while the kicking term arises from a time periodic standing wave laser field. Obviously such examples require a proper quantum mechanical treatment of the Hamiltonian (1), the corresponding Schroedinger equation being (once expressed in dimensionless variables $\tilde{t}=\omega_{0} t$ and $\left.\tilde{x}=\sqrt{m_{0} \omega_{0} / \hbar} x\right)$

$$
i \frac{\partial}{\partial \tilde{t}} \psi=\left(-\frac{1}{2} \frac{\partial^{2}}{\partial \tilde{x}^{2}}+\frac{1}{2} \tilde{x}^{2}+\sigma \cos (\xi \tilde{x}) \delta_{T}(\tilde{t})\right) \psi
$$

so that quantum dynamics depends upon three parameters

$$
\sigma=\frac{\varepsilon}{\hbar} \quad \xi=k_{0} \sqrt{\frac{\hbar}{m_{0} \omega_{0}}} \quad T=T_{0} \omega_{0}
$$

Once again the behavior is quite sensitive to number theoretic properties of $T$ : in particular the crystal cases [10] $T=2 \pi / q$ with $q \in\{1,2,3,4,6\}$ admit a one-parameter group of commuting generalized translations commuting with the hamiltonian (exceptional parameter values 10 may also lead to two-parameter groups): the corresponding dynamical behavior is diffusive (or ballistic in the exceptional cases). We remark that the $q=4$ case corresponds to the (symmetric) kicked Harper model [17]. Outside resonant parameter values there are indication of a localization-delocalization transition for resonant-non crystal cases [11, while the simulations reported in 10 for non-resonant cases suggest dynamical localization 18 (however we observed a delocalization transition in the irrational case too).

Recently it has been suggested [19] how the widespread interest and experimental activity in Bose-Einstein condensation 20] makes it natural to study the effect on Gross-Pitaevskii nonlinearities [21] on the kicked oscillator (thus turning it into a model of a trapped condensate under a laser field in the spirit of [16]). The 
Gross-Pitaevskii nonlinear correction to the Schroedinger equation is of the form $u|\psi|^{2} \psi$, where the coefficient $u$ is of the same sign of the scattering length 22] (we will mainly deal with a positive $u$ in what follows): using the same rescaling in dimensionless variables mentioned in the quantum case the equation reads

$$
i \frac{\partial}{\partial \tilde{t}} \psi=\left(-\frac{1}{2} \frac{\partial}{\partial \tilde{x}^{2}}+\frac{1}{2} \tilde{x}^{2}+\sigma \cos (\xi \tilde{x}) \delta_{T}(\tilde{t})+v|\psi|^{2}\right) \psi
$$

where now

$$
v=\frac{u}{\hbar} \sqrt{\frac{m_{0}}{\hbar \omega_{0}}}
$$

Even if the cubic nonlinearity acts like an effective repulsive potential, the main observation in 19, as regards the dynamical effect of the Gross-Pitaevskii nonlinearity in a crystal $q=6$ case, has been its tendency to oppose quantum spreading: it was suggested that this is due to a breakup of quantum symmetries for nonzero nonlinearity. Before presenting the results of our simulations we have to mention that nonlinearity effects have also been considered a few years ago for the kicked rotator 23.24 (for a cubic nonlinearity of opposite sign). Here the scenario is quite different: when the nonlinearity is absent the system exhibits quantum dynamical localization: a sufficiently strong nonlinearity may then induce chaotic transitions between localized modes, leading to (subdiffusive) delocalization.

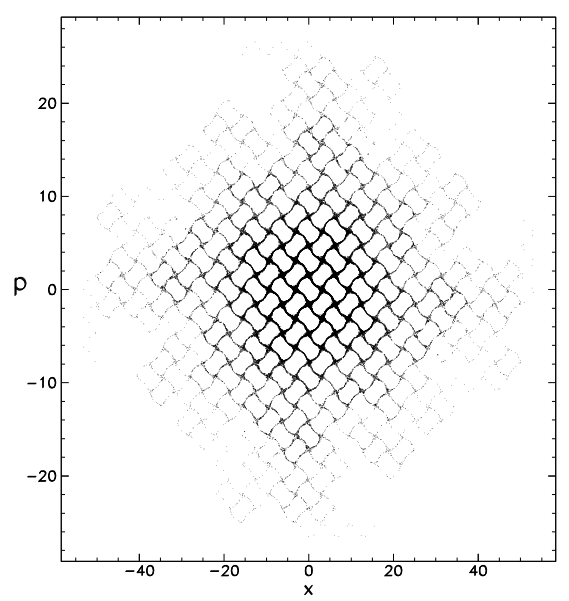

FIG. 1. Classical transport along the stochastic web.

To investigate the effect of the nonlinearity, we studied the equation (5) in two different regimes: a crystal $(q=4)$ case, and an irrational case $(T=\pi /(\sqrt{ } 5+1))$. The evolution of the the kicked oscillator is conveniently studied by using a discretized propagator [10]:

$$
\psi\left(x^{\prime}, t^{\prime}\right)=\int d x \mathcal{G}\left(x^{\prime}, x ; t\right) \psi(x, 0)
$$

where

$\mathcal{G}\left(x^{\prime}, x ; t\right)=C \exp \left\{\frac{i m_{0} \omega_{0}}{2 \hbar \sin \left(\omega_{0} t\right)}\left(\left(x^{2}+x^{\prime 2}\right) \cos \left(\omega_{0} t\right)-2 x x^{\prime}\right)\right\}$

and once the discretized positions $x_{i}=(i-N / 2) \Delta_{x}$ are introduced we have that the propagator $\mathcal{G}$ is unitary if we put

$$
\Delta_{x}=\left(\frac{2 \pi \hbar \sin \left(\omega_{0} t\right)}{m_{0} \omega_{0} N}\right)^{1 / 2}
$$

In the coordinate representation the action of kicks is multiplicative on the wave function.

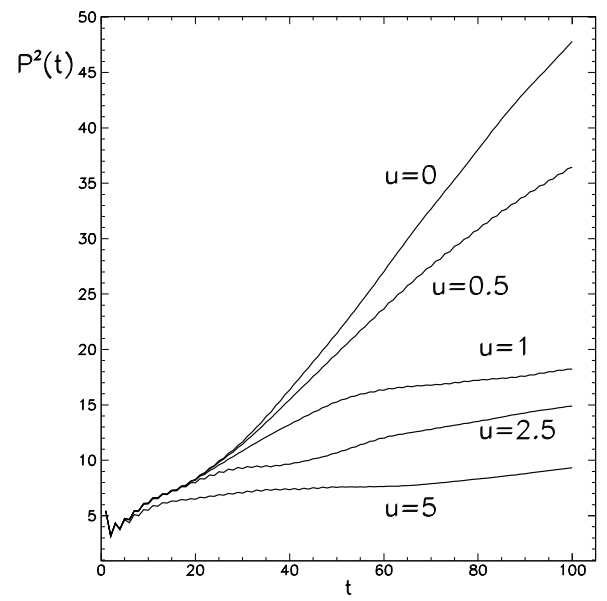

FIG. 2. Quantum transport ( $q=4$ one parameter symmetry group). See the text for details.

Simulation of quantum evolution is considerably more complicated once we introduce a nonzero nonlinearity: to propagate the wave function between kicks we separate the time independent part of the hamiltonian into the oscillator and the nonlinear part, and use the lowest order split method [25] (this typically requires using ten time steps between consecutive kicks in order to get stable results). As initial state we consider the ground state of the GP equation (without kicks) shifted in in the chaotic region nearest to the origin. The ground states for different values of the nonlinearity parameter are obtained by evolving an eigenstate of the quantum harmonic oscillator under the imaginary time Gross-Pitaevskii equation 26.

The first case we take into account is a crystal $q=4$ example. In fig. (1) we show the classical phase picture, exhibiting unbounded transport along the stochastic web. We put $T=\pi / 2$ (and $\omega_{0}$ fixed in such a way that 
generalized translations form a one parameter group of symmetries), $\epsilon=0.7$ (all others linear quantum parameters are fixed by taking $\hbar=1$ and $\xi=\sqrt{ } 2$ : we will adopt this choice for other examples too).

This case is characterized by a one-parameter group of simmetries (generalized phase space translations) and thus the quantum case is expected [10] to exhibit a diffusive momentum spreading (the evolution corresponds of the upper line in fig. (2)): the effect of nonlinearity is considering by taking $u=0.5,1,2.5$ and 5 : the corresponding curves are shown in fig. (2). Such simulations are performed by using a $N=2^{14}$ discretization of the position variable (which has been checked to provide a reliable choice up to the considered evolution time, by comparing the results with a simulation with twice the number of points). To smooth out oscillations in the evolving patterns we plot the integrated second moment

$$
P^{2}(t)=\frac{1}{t} \sum_{k=0}^{t-1}\left\langle\left(p_{k}-p_{0}\right)^{2}\right\rangle
$$

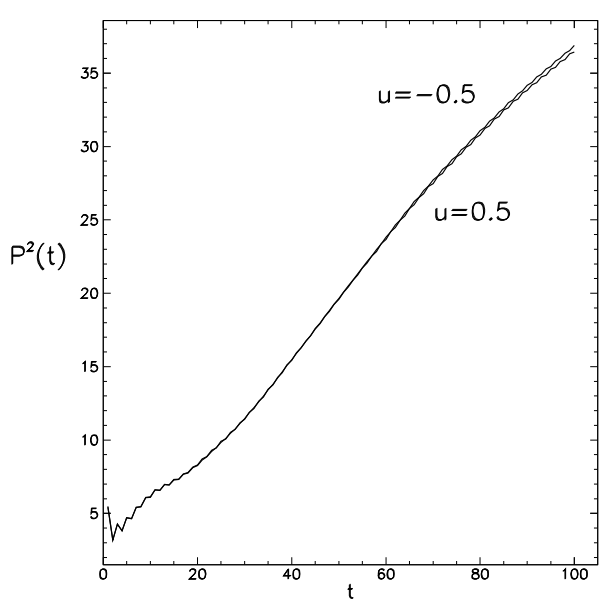

FIG. 3. $P^{2}(t)$, for $u=+0.5$ and $u=-0.5$, and same conditions as fig. (2).

The qualitative features confirm the observation in [19], namely that the most striking effect of the nonlinearity is to oppose quantum delocalization. This has been claimed to be due to symmetry breaking effects of the nonlinearity, inhibiting transport along delocalized Floquet states. We remark that also at a classical level related features have been observed: if noise is added to the kicked Harper map, transport along the stochastic web is slowed down 27.

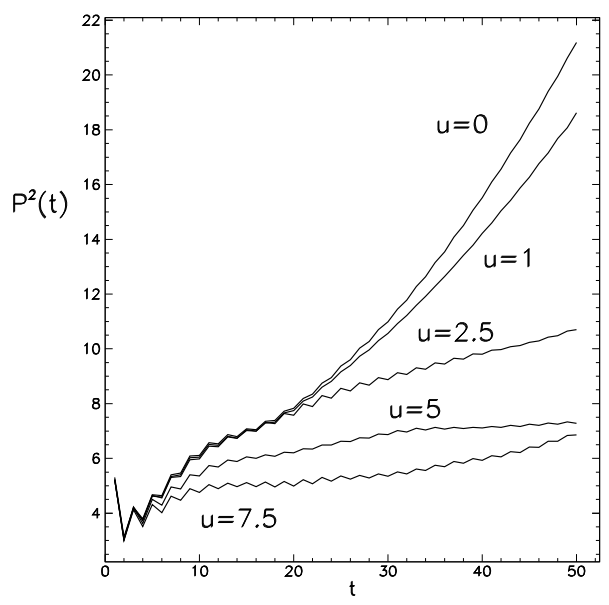

FIG. 4. Quantum transport ( $q=4$ two-parameter symmetry group) group, $\epsilon=0.7$. The configuration space has been discretized with $2^{12}$ points.

In principle a positive nonlinearity acts like a repulsive potential, but here the symmetry breaking effect is not related to the sign of the effective potential, as we see from fig.(3), where it is shown how the sign of the nonlinearity has a tiny effect on momentum spreading.

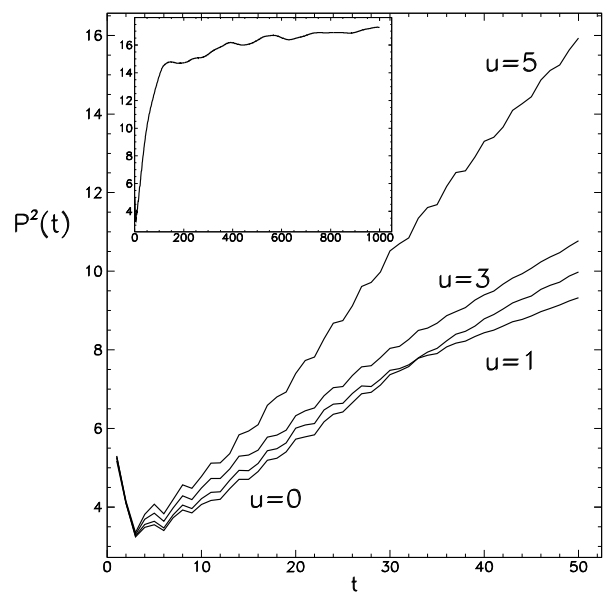

FIG. 5. Quantum transport irrational case. Discretization is over $2^{14}$ points. The inset shows the kicked oscillator case on a longer time scale.

If such a view is correct, the effect must equally appear in the resonant crystal case, where a group of twoparameter symmetries leads to ballistic transport [10]: such an effect is indeed evident even for short times, see fig. (4): after a characteristic time scale, which shrinks as nonlinearity increases, the deviation from the kicked 
oscillator case is more and more marked as $u$ increases.

A priori the situation is different when we consider the oscillator outside the crystal regime: to this end we analyzed the case in which $\epsilon=1.4$ and $T=\pi /(\sqrt{ } 5+1)$. In this case the kicked oscillator displays dynamical localization: the striking observation is that here the nonlinearity acts in an opposite way, enhancing the quantum delocalization, see fig. (5). So, when symmetries are not present in the quantum case, nonlinearity seems to play a completely different role. This is at least in qualitative agreement to what happens to the kicked rotator evolving under a nonlinear Schroedinger equation, or even when noise is superimposed to the quantum evolution 28]. We have checked that the same happens even for higher values of $\epsilon$, when the oscillator undergoes a delocalization transition.

In conclusion, we have analyzed how nonlinearity influences a complex, and physically relevant quantum system, the kicked harmonic oscillator. We provide evidences that, at least at moderate times, it opposes quantum diffusion when transport is linked to symmetry properties of the linear hamiltonian, while it may lead to diffusion enhancement when no symmetry breaking occurs.

This work was partially supported by the PRIN-2000 project Chaos and Localization in classical and quantum systems, and the EU contract QTRANS network (Quantum Transport on an Atomic Scale). We thank E. Arimondo and J.H. Müller for pointing ref. [19] to our attention.

[1] G. Casati and B.V. Chirikov, Quantum chaos: between order and disorder, (Cambridge University Press, Cambridge, 1995) and references therein.

[2] G. Casati, B.V. Chirikov, J. Ford and F.M. Izrailev, in Stochastic Behaviour in Classical and Quantum Hamiltonian Systems, Springer Lect.Notes Phys. 93, 334 (1979).

[3] A detailed review of the properties of such a system is contained in F.M. Izrailev, Phys.Rep. 196, 299 (1990).

[4] B.V. Chirikov, Phys.Rep. 52, 264 (1979).

[5] See, for instance, A.B. Rechester and R.B. White, Phys.Rev.Lett. 44, 1586 (1980) and I. Dana, N. Murray and I. Percival, Phys.Rev.Lett. 62, 233 (1989).

[6] An asymptotic localized regime is also found for different classical control parameter values, where transport is of anomalous nature [7].

[7] R. Artuso and M. Rusconi, Phys.Rev. E64, 015204(R) (2001).

[8] F.M. Izrailev and D.L. Shepelyansky, Dokl.Akad.Nauk SSSR 249, 1103 (1979) [Sov.Phys.-Dokl. 24, 996 (1979)].

[9] G.P. Berman, V. Yu. Rubaev and G.M. Zaslavsky, Nonlinearity 4, 543 (1991).

[10] F. Borgonovi and L. Rebuzzini, Phys.Rev. E52, 2302 (1995).

[11] D. Shepelyansky and C. Sire, Europhys.Lett. 20, 95
(1992).

[12] G.M. Zaslavsky, M. Yu. Zakharov, R.Z. Sagdeev, D.A. Usikov and A.A. Chernikov, Zh.Eksp.Teor.Fiz. 91, 500 (1986); A.A. Chernikov, R.Z. Sagdeev, D.A. Usikov, M.Yu. Zakharov and G.M. Zaslavsky, Nature 326, 559 (1987).

[13] This is possible as the model does not satisfy the requirements of Kolmogorov-Arnold-Moser theorem.

[14] A.A. Chernikov, R.Z. Sagdeev and G.M. Zaslavsky, Physica D33, 65 (1988).

[15] T.M. Fromhold, A.A. Krokhin, C.R. Tench, S. Bujkiewicz, P.B. Wilkinson, F.W. Sheard and L. Eaves, Phys.Rev.Lett. 87, 046803 (2001).

[16] S.A. Gardiner, J.I. Cirac and P. Zoller, Phys.Rev.Lett. 79, 4790 (1997).

[17] See R. Artuso, F. Borgonovi, G. Casati, I. Guarneri and L. Rebuzzini, Int.J.Mod.Phys. B8, 207 (1994), and references therein.

[18] We remark that, from a point of view of numerical simulations, the kicked harmonic oscillator is harder to treat than the kicked rotator, the natural phase space being the plane $\mathbf{R}^{2}$, instead of the cylinder $\mathbf{R} \times S$, induced by the angle character of the coordinate variable.

[19] S.A. Gardiner, D. Jaksch, R. Dum, J.I. Cirac and P. Zoller, Phys.Rev. A62, 023612 (2000).

[20] See, for instance the reviews by W. Ketterle et al. and E. Cornell et al in Bose-Einstein Condensation in atomic gases, edited by M. Inguscio, S. Stringari and C. Wieman (IOS Press, Amsterdam, 1999).

[21] See F. Dalfovo, S. Giorgini, L.P. Pitaevskii and S. Stringari, Rev.Mod.Phys. 71, 463 (1999) and references therein.

[22] We are considering wave functions normalized to unity, so that $u$ may be espressed as $4 \pi N \hbar^{2} a_{s} / m_{0}$, where $N$ is the number of particles in the condensate, and $a_{s}$ is the s-wave scattering length.

[23] F. Benvenuto, G. Casati, A.S. Pikovsky and D.L. Shepelyansky, Phys.Rev. A44, R3423 (1991).

[24] D.L. Shepelyansky, Phys.Rev.Lett. 70, 1787 (1993); Physica D86, 45 (1995).

[25] See for instance A.D. Bandrauk and H. Shen, J.Chem.Phys. 99, 1185 (1993); J.Phys. A27, 7147 (1994).

[26] R. Baer, Phys.Rev. A62, 063810 (2000).

[27] A.J. Lichtenberg and B.P. Wood, Phys.Rev.Lett. 62,2213 (1989); G. Park and C.S. Chang, Phys.Rev. E63, 066213 (2001).

[28] E. Ott, T.M. Antonsen, Jr. and J.D. Hanson, Phys.Rev.Lett. 53, 2187 (1984). 